TRANSACTIONS OF THE

AMERICAN MATHEMATICAL SOCIETY

Volume 348, Number 11, November 1996

\title{
CURVATURE INVARIANTS, DIFFERENTIAL OPERATORS AND LOCAL HOMOGENEITY
}

\author{
FRIEDBERT PRÜFER, FRANCO TRICERRI ${ }^{\dagger}$, AND LIEVEN VANHECKE
}

\begin{abstract}
We first prove that a Riemannian manifold $(M, g)$ with globally constant additive Weyl invariants is locally homogeneous. Then we use this result to show that a manifold $(M, g)$ whose Laplacian commutes with all invariant differential operators is a locally homogeneous space.
\end{abstract}

\section{INTRODUCTION}

A Riemannian manifold $(M, g)$ is said to be locally homogeneous if its pseudogroup of local isometries acts transitively on $(M, g)$. Of course, every homogeneous space is locally homogeneous, but the converse does not hold. Moreover, there exist examples of locally homogeneous spaces which are not locally isometric to a globally homogeneous space (see [10], [18], [21] for explicit examples).

There are several special classes of Riemannian manifolds where only locally homogeneous examples are known. For example, this happens for the classes of harmonic spaces, spaces with volume-preserving geodesic symmetries, and ballhomogeneous spaces. It remains an interesting open problem if there exist examples in these classes which are not locally homogeneous. (See [3], [11], [12], [22] for a survey and further references about these spaces.)

In order to attack this problem, it is important to have at our disposal a series of necessary and sufficient conditions for local homogeneity. The main purpose of this paper is to provide such a criterion. It stems from the fact that in the study of the special Riemannian manifolds cited above, and in many other places, the scalar curvature invariants play a fundamental role. These invariants are by definition polynomials in the components of the Riemannian curvature tensor and its covariant derivatives which do not depend on the choice of orthonormal basis of the tangent space at each point. It is clear that such invariants are global constants on a locally homogeneous space. We will prove that the converse holds. In fact, we will show more, and prove that the converse also holds when we restrict to the special set formed by the additive Weyl invariants. (See below for the definition.) Therefore, we study the Weyl invariants in more detail and prove that each of them can be represented as a product of additive ones.

Received by the editors September 26, 1995.

1991 Mathematics Subject Classification. Primary 53C20, 53C25, 53C30.

Key words and phrases. Curvature invariants, locally homogeneous spaces, Laplacian, invariant differential operators, commutativity, spaces with volume-preserving geodesic symmetries.

${ }^{\dagger}$ To our deep sorrow F. Tricceri died in an airplane crash in China on the sixth of June 1995. His contribution to this paper was essential. 
The second part of the paper contains an application and concerns differential operators on a Riemannian manifold which are invariant under isometries. It originates from the fact that an $(M, g)$ has volume-preserving geodesic symmetries if its Laplacian $\Delta$ commutes with a whole class of special invariant differential operators, namely the class of Euclidean Laplacians. In this paper we will prove that $(M, g)$ is locally homogeneous if $\Delta$ commutes with a special set of invariant second order differential operators constructed by means of additive Weyl invariants. As a consequence of this result, it turns out that a Riemannian manifold whose Laplacian commutes with all invariant differential operators is a locally homogeneous space with volume-preserving geodesic symmetries.

\section{Curvature invariants And Local homogeneity}

Let $(M, g)$ be an $n$-dimensional, smooth, connected Riemannian manifold and let $O M=(O M, \pi, M, O(n))$ be the bundle of orthonormal frames over $(M, g)$. An element $u=\left(q ; u_{1}, \ldots, u_{n}\right)$ of $O M$ induces an isometry, also denoted by $u$, of $V=\mathbb{R}^{n}$ on $T_{q} M$ defined by

$$
u(\xi)=u\left(\xi^{1}, \ldots, \xi^{n}\right)=\sum_{i=1}^{n} \xi^{i} u_{i} .
$$

Let $\nabla$ denote the Levi-Civita connection and $R$ the corresponding Riemannian curvature tensor of $(M, g)$. For each $s \geq 0$, the tensor field $\nabla^{s} R$ defines a map $K^{s}$ from $O M$ to $\otimes^{s+4} V^{*}$ given by

$$
K^{s}(u)\left(\xi_{1}, \ldots, \xi_{s+4}\right)=\nabla^{s} R_{\mid \pi(u)}\left(u \xi_{1}, \ldots, u \xi_{s+4}\right),
$$

where $\xi_{1}, \ldots, \xi_{s+4} \in V$.

The orthogonal group $O(n)$ acts on the left on the tensor product $\otimes^{\ell} V^{*}$ by the rule

$$
(a T)\left(\xi_{1}, \ldots, \xi_{\ell}\right)=T\left(a^{-1} \xi_{1}, \ldots, a^{-1} \xi_{\ell}\right)
$$

and it is easy to verify that $K^{s}$ is an equivariant map with respect to the actions of $O(n)$ on $O M$ and on $\otimes^{s+4} V^{*}$, that is,

$$
K^{s}(u a)=a^{-1} K^{s}(u)
$$

for all $u \in O M$ and $a \in O(n)$.

Following [17] (see also [13]) we consider now the map $\phi_{m}: O M \rightarrow W_{m}=$ $\bigoplus_{s=0}^{m}\left(\otimes^{s+4} V^{*}\right)$ defined by

$$
\phi_{m}(u)=\sum_{s=0}^{m} K^{s}(u) .
$$

Then $\phi_{m}$ is also equivariant, that is,

$$
\phi_{m}(u a)=a^{-1} \phi_{m}(u)
$$

for all $u \in O M, a \in O(n)$.

Next, let $\mathbb{R}[W]^{O(n)}$ denote the $\mathbb{R}$-algebra of $O(n)$-invariant polynomial functions on a representation space $W$. If $p(w)$ is such a polynomial for $W=W_{m}$, then $p \circ \phi_{m}$ is constant along the fibers of $O M$ :

$$
\left(p \circ \phi_{m}\right)(u a)=p\left(a^{-1} \phi_{m}(u)\right)=p\left(\phi_{m}(u)\right)=\left(p \circ \phi_{m}\right)(u) .
$$


Therefore, there exists a unique $C^{\infty}$ map $f: M \rightarrow \mathbb{R}$ such that

$$
f \circ \pi=p \circ \phi_{m},
$$

that is, the following diagram commutes:

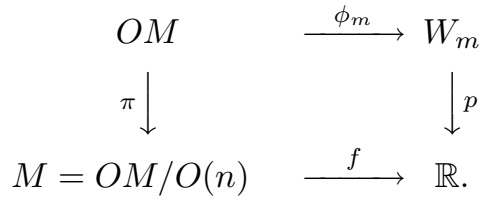

We call $f$ a scalar curvature invariant of order $m$ of $(M, g)$. Note that $f$ is an $O(n)$-invariant polynomial in the components of $R, \nabla R, \ldots, \nabla^{m} R$ with respect to some fixed orthonormal basis of each tangent space $T_{q} M, q \in M$.

Since $O(n)$ is compact, we have the following result of Hilbert [14], [15], [24] :

Proposition 2.1. The algebra $\mathbb{R}[W]^{O(n)}$ is finitely generated. If $p_{1}, \ldots, p_{t}$ are generators, then the polynomial map $p: W \rightarrow \mathbb{R}^{t}, w \mapsto p(w)=\left(p_{1}(w), \ldots, p_{t}(w)\right)$ separates the orbits of $O(n)$ in $W$.

This means that $w$ and $w^{\prime}$ are in the same orbit if and only if $p(w)=p\left(w^{\prime}\right)$.

As an immediate consequence we have:

Corollary 2.2. Let $(M, g)$ be a Riemannian manifold such that all the scalar curvature invariants of order $m$ are global constants. Then $\phi_{m}(O M)$ is contained in a single $O(n)$-orbit in $W_{m}$.

Proof. $p \circ \phi_{m}$ is constant on the fibers in $O M$, and (2.1) shows that it does not depend on the points $q$ of $M$, either.

Note that for $m=0$ we obtain that $(M, g)$ is curvature homogeneous.

Now, we prove

Theorem 2.3. Let $(M, g)$ be a Riemannian manifold of dimension $n$ such that all scalar curvature invariants of order $m+1$ with $m+1 \leq \frac{n(n-1)}{2}$ are constant. Then $(M, g)$ is locally homogeneous. Moreover, $(M, g)$ is uniquely determined by these curvature invariants up to a local isometry.

Proof. $\phi_{m+1}$ is contained in a single orbit for all $m+1 \leq \frac{n(n-1)}{2}$. This implies that $(M, g)$ is infinitesimally homogeneous, since the Singer integer $k(p)$ at a point $p \in M$ is not greater than $\frac{1}{2} n(n-1)-1$ [17]. As a consequence, $(M, g)$ is locally homogeneous (see [17] for the global homogeneous case and [13] for a local version). The last statement follows from Theorem 2.5 in [13].

Note that the converse is trivially true. Hence we obtain a criterion for local homogeneity. Note also that another estimate for the Singer integer is given in $[8$, p. 165] without proof, namely $k(p)+1<\frac{3}{2} n$.

\section{WEYL AND ADDITIVE WEYL INVARIANTS}

Now we focus our attention on special scalar curvature invariants. It follows from Weyl's theory of invariants [24] (see also [1, p. 76]) that the invariant polynomials are contractions in the components of the curvature tensor and its covariant 
derivatives, and hence, linear combinations of Weyl invariants. Here a (non-trivial) Weyl invariant $W$ (i.e., a product of traces [1]) is given by

$$
W=\operatorname{tr}\left(\nabla^{\ell_{1}} R \otimes \cdots \otimes \nabla^{\ell_{\nu}} R\right)
$$

$\left(\ell_{j} \in \mathbb{N}_{0}, j=1, \ldots, \nu ; \nu \in \mathbb{N}\right), \nabla^{0} R=R$ and tr means the complete trace with respect to some permutation of the indices.

Obviously, any Weyl invariant $W$ given by (3.1) is defined for every Riemannian manifold $(M, g)$. For two Weyl invariants $W_{1}$ and $W_{2}$ we say $W_{1}=W_{2}$ if and only if $W_{1}(q)=W_{2}(q)$ for each $q \in M$ and every $(M, g)$. Now, let $\lambda \in \mathbb{R}_{+}$. Then we have $W(\lambda g)=\lambda^{-\mu} W(g)$, and we call $\mu \in \mathbb{N}$ the degree of $W$. So, for $W$ in (3.1) we get $\operatorname{deg} W=\frac{1}{2}\left(\ell_{1}+\cdots+\ell_{\nu}\right)+\nu$. Then we have for $W=W_{1} W_{2}$ that $\operatorname{deg} W=$ $\operatorname{deg} W_{1}+\operatorname{deg} W_{2}$.

Clearly, we have

Corollary 3.1. Let $(M, g)$ be a Riemannian manifold with globally constant Weyl invariants. Then $(M, g)$ is locally homogeneous.

Now, as usual, we write $T_{i_{1} \ldots i_{k}}=T\left(e_{i_{1}}, \ldots, e_{i_{k}}\right)$ for a tensor of type $(0, k)$ with respect to an orthonormal basis $\left(e_{1}, \ldots, e_{n}\right)$ of $T_{q} M$ at $q \in M$. Then a Weyl invariant $W$ at $q$ may be written, with respect to this frame, in the following form:

$$
W=\sum_{\left(a_{1}\right)_{1}, \ldots,\left(a_{\nu}\right)_{\ell_{\nu}+4}} \sigma\left(\delta^{A_{1} \cdots A_{\nu}}\right)\left(\nabla^{\ell_{1}} R\right)_{A_{1}} \cdots\left(\nabla^{\ell_{\nu}} R\right)_{A_{\nu}},
$$

where $A_{\kappa}=\left(\left(a_{\kappa}\right)_{1}, \ldots,\left(a_{\kappa}\right)_{\ell_{\kappa}+4}\right),\left(\nabla^{\ell_{\kappa}} R\right)_{A_{\kappa}}=\nabla_{\left(a_{\kappa}\right)_{1} \cdots\left(a_{\kappa}\right)_{\ell_{\kappa}}}^{\ell_{\kappa}} R_{\left(a_{\kappa}\right)_{\ell_{\kappa}+1} \cdots\left(a_{\kappa}\right)_{\ell_{\kappa}+4}}, \kappa=$ $1, \ldots, \nu$, and $\sigma\left(\delta^{A_{1} \cdots A_{\nu}}\right)=\delta^{\sigma\left(a_{1}\right)_{1} \sigma\left(a_{1}\right)_{2}} \cdots \delta^{\sigma\left(a_{\nu}\right) \ell_{\nu}+3 \sigma\left(a_{\nu}\right) \ell_{\nu}+4}, \delta^{i j}$ being the Kronecker symbol and $\sigma$ a permutation of the indices $\left(a_{1}\right)_{1}, \ldots,\left(a_{\nu}\right)_{\ell_{\nu}+4}$.

Then we have

Lemma 3.2. Let $W$ be a Weyl invariant of degree $\mu$. Then $W=W_{1} W_{2}$, where $W_{i}, i=1,2$, are Weyl invariants of degree $\mu_{i}<\mu$, if and only if there exists a permutation $\sigma$ in the expression (3.2) of $W$ which restricts to a permutation of a proper subset $\left\{A_{\alpha_{1}}, \ldots, A_{\alpha_{r}}\right\}$ of $\left\{A_{1}, \ldots, A_{\nu}\right\}$.

Proof. Let $W=W_{1} W_{2}$ with degree $W_{i}<\mu$. Then the result is obvious.

Conversely, suppose we can restrict $\sigma$ in such a way. Put $W_{1}=\operatorname{tr} \nabla^{\ell_{\alpha_{1}}} R \otimes$ $\cdots \otimes \nabla^{\ell_{\alpha_{r}}} R$ with the permutation $\sigma_{1}$ in (3.2) given by the restriction of $\sigma$ to $\left\{A_{\alpha_{1}}, \ldots, A_{\alpha_{\nu}}\right\}$. Then the restriction of $\sigma$ to $\left\{A_{\beta_{1}}, \ldots, A_{\beta_{s}}\right\}=\left\{A_{1}, \ldots, A_{\nu}\right\} \backslash\left\{A_{\alpha_{1}}, \ldots\right.$, $\left.A_{\alpha_{r}}\right\}$ is also a permutation. Using $\sigma_{2}$ in the expression (3.2), we obtain a second Weyl invariant $W_{2}=\operatorname{tr} \nabla^{\ell_{\beta_{1}}} R \otimes \cdots \otimes \nabla^{\ell_{\beta_{s}}} R$, and obviously $W=W_{1} W_{2}$. Since $\mu=\mu_{1}+\mu_{2}$ we have $\mu_{i}<\mu, i=1,2$, and the result follows.

Next, a Weyl invariant $W$ is said to be additive if for all $M=M_{1} \times M_{2}$ we have

$$
W_{M}\left(q_{1}, q_{2}\right)=W_{M_{1}}\left(q_{1}\right)+W_{M_{2}}\left(q_{2}\right),
$$

where $\left(q_{1}, q_{2}\right) \in M_{1} \times M_{2}$ and where $W_{M}, W_{M_{1}}$ and $W_{M_{2}}$ are the corresponding Weyl invariants of $W$ for $M, M_{1}$ and $M_{2}$, respectively. For example, it is well-known that the scalar curvature $\tau$ is an additive Weyl invariant, but $\tau^{2}$ is not additive.

Now we prove

Theorem 3.3. Every Weyl invariant is a product of additive Weyl invariants. 
Proof. Let $\left(M=M_{1} \times M_{2}, g\right)$ be the Riemannian product of $\left(M_{1}, g_{1}\right)$ and $\left(M_{2}, g_{2}\right)$ and denote by $\nabla, \stackrel{(1)}{\nabla}, \stackrel{(2)}{\nabla}$ their respective Levi-Civita connections and by $R \stackrel{(1)}{R} \stackrel{(2)}{R}$ the corresponding curvature tensors. Let $\operatorname{dim} M_{1}=n_{1}$ and $\operatorname{dim} M_{2}=n_{2}$, and put $I_{1}=\left\{1, \ldots, n_{1}\right\}, I_{2}=\left\{n_{1}+1, \ldots, n_{1}+n_{2}\right\}$. With a similar notation as for (3.2) we have

$$
\left(\nabla^{\ell} R\right)_{A_{\ell}}(q)= \begin{cases}\left({ }^{\ell} \stackrel{(1)}{R}^{(1)}\right)_{A_{\ell}}\left(q_{1}\right) & \text { for } A_{\ell} \subseteq I_{1} \times \cdots \times I_{1}, \\ \left(\nabla^{\ell} \stackrel{(2)}{R}\right)_{A_{\ell}}\left(q_{2}\right) & \text { for } A_{\ell} \subseteq I_{2} \times \cdots \times I_{2}, \\ 0 & \text { otherwise, }\end{cases}
$$

where $q_{1} \in M_{1}, q_{2} \in M_{2}$ and $\left\{a_{1}, \ldots, a_{n_{1}}\right\}$, respectively $\left\{a_{n_{1}+1}, \ldots, a_{n_{1}+n_{2}}\right\}$, is an orthonormal basis of $T_{q_{1}} M$, respectively $T_{q_{2}} M_{2}$.

For a Weyl invariant $W$ we have the following two possibilities:

(i) $W=V_{1} V_{2}$ where $\mu_{i}=$ degree $V_{i}<\mu=$ degree $W, i=1,2$;

(ii) $W$ cannot be decomposed as such a product.

In case (ii), Lemma 3.2 yields that the possible permutation $\sigma$ in the representation (3.2) of $W$ cannot restrict to a proper subset of $\left(A_{1}, \ldots, A_{\nu}\right)$. But then it follows at once from (3.2) and (3.3) that in the corresponding sum we have only terms with indices belonging either to $I_{1}$ or to $I_{2}$, that is, $W=W_{1}+W_{2}$ and $W$ is additive. In case (i) we may make a similar consideration for $V_{1}$ and $V_{2}$ as for $W$. After a finite number of steps we get $W=\bar{V}_{1} \cdots \bar{V}_{r}, \mu=\mu_{1}+\cdots+\mu_{r}, 1 \leq \mu_{i}<\mu$, where each $\bar{V}_{\alpha}, \alpha=1, \ldots, r$, cannot be decomposed further. Then, as in case (ii), it follows that each $\bar{V}_{\alpha}$ is additive, and so the proof is complete.

From Corollary 3.1 and Theorem 3.3 we get at once

Corollary 3.4. Let $(M, g)$ be a Riemannian manifold such that each additive Weyl invariant is globally constant. Then $(M, g)$ is locally homogeneous.

Finally, we give a criterion for the additivity of a Weyl invariant by means of the irreducibility of the polynomials $p \in \mathbb{R}[W]^{O(n)}$. We start with

Lemma 3.5. Let $f$ be a scalar curvature invariant. Then $f=f_{1} f_{2}$ with scalar curvature invariants $f_{i}, i=1,2$, if and only if there exists a reducible polynomial for $f$ in the representation (2.1).

Proof. First, let $f \circ \pi=p \circ \phi_{m}$ with $p=p_{1} p_{2}$. This gives

$$
f \circ \pi=\left(p_{1} p_{2}\right) \circ \phi_{m}=\left(p_{1} \circ \phi_{m}\right)\left(p_{2} \circ \phi_{m}\right)=\left(f_{1} \circ \pi\right)\left(f_{2} \circ \pi\right)=\left(f_{1} f_{2}\right) \circ \pi
$$

and hence $f=f_{1} f_{2}$.

Conversely, let $f=f_{1} f_{2}$. Put $f_{1} \circ \pi=p_{1} \circ \phi_{m_{1}}, f_{2} \circ \pi=p_{2} \circ \phi_{m_{2}}$. Then we can find suitable $\tilde{p}_{1}, \tilde{p}_{2}, \tilde{m}$ such that $f_{1} \circ \pi=\tilde{p}_{1} \circ \phi_{\tilde{m}}, f_{2} \circ \pi=\tilde{p}_{2} \circ \phi_{\tilde{m}}$; and we get

$$
f \circ \pi=\left(f_{1} f_{2}\right) \circ \pi=\left(f_{1} \circ \pi\right)\left(f_{2} \circ \pi\right)=\left(\tilde{p}_{1} \circ \phi_{\tilde{m}}\right)\left(\tilde{p}_{2} \circ \phi_{\tilde{m}}\right)=\left(\tilde{p}_{1} \tilde{p}_{2}\right) \circ \phi_{\tilde{m}} .
$$

Therefore we can choose $p=\tilde{p}_{1} \tilde{p}_{2}$ for $f$ in the representation (2.1).

From this we get

Corollary 3.6. Let $W$ be a Weyl invariant. If all possible polynomials $p$ in $W \circ \pi=$ $p \circ \phi_{m}$ are irreducible, then $W$ is additive. 
Proof. The hypothesis implies that $W$ cannot be equal to $W_{1} W_{2}$ where $W_{1}$ and $W_{2}$ are Weyl invariants. Then the result follows from Theorem 3.3.

To prove the converse of this corollary, and hence to obtain the required criterion, we shall use three lemmas which we consider first.

Lemma 3.7. Let $W=\sum_{i=1}^{\ell} a_{i} W_{i}$ be an additive Weyl invariant, where the $a_{i}$ are all non-zero and the $W_{i}$ are linearly independent Weyl invariants. Then each $W_{i}$ is additive.

Proof. Following Theorem 3.3 we put $W_{i}=\prod_{j=1}^{\kappa_{i}} U_{i j}, i=1, \ldots, \ell$, where each $U_{i j}$ is an additive Weyl invariant. Hence, we have

$$
W=\sum_{i=1}^{\ell} a_{i} \prod_{j=1}^{\kappa_{i}} U_{i j}
$$

Now, let $(q, q) \in M \times M$ where $M$ is an arbitrary Riemannian manifold. Then we have

$$
W_{M \times M}(q, q)=2 W_{M}(q)=\sum_{i=1}^{\ell} a_{i} \prod_{j=1}^{\kappa_{i}} U_{i j M \times M}(q, q)=\sum_{i=1}^{\ell} a_{i} 2^{\kappa_{i}} \prod_{j=1}^{\kappa_{i}} U_{i j M}(q),
$$

and thus

$$
W=\sum_{i=1}^{\ell} a_{i} 2^{\kappa_{i}-1} \prod_{j=1}^{\kappa_{i}} U_{i j}
$$

or

$$
\sum_{i=1}^{\ell} a_{i}\left(2^{\kappa_{i}-1}-1\right) W_{i}=0
$$

The hypotheses now imply that $\kappa_{i}=1$ for $i=1, \ldots, \ell$, and hence $W_{i}=U_{i 1}$, where $U_{i 1}$ is additive.

Lemma 3.8. Let $W$ be a non-vanishing additive Weyl invariant such that $W=$ $\prod_{j=1}^{\kappa} U_{j}$ where each $U_{j}$ is a Weyl invariant. Then $\kappa=1$.

Proof. It follows from Theorem 3.3 that $U_{j}=\prod_{i=1}^{\kappa_{j}} U_{j i}$ where each $U_{j i}$ is an additive Weyl invariant. Thus, we have

$$
W=\prod_{j=1}^{\kappa} U_{j 1} \cdots U_{j \kappa_{j}}
$$

Next, using the same procedure as in the proof of Lemma 3.7, we now get

$$
2 W=2^{\sum_{j=1}^{\kappa} \kappa_{j}} W
$$

and so, $\sum_{j=1}^{\kappa} \kappa_{j}=1$. The result now follows at once since $\kappa_{j} \geq 1, j=1, \ldots, \kappa$.

Lemma 3.9. Let $W=\prod_{i=1}^{k} f_{i}$ be an additive Weyl invariant where each $f_{i}, i=$ $1, \ldots, k$, is a scalar curvature invariant. Then $\kappa=1$. 
Proof. Since every scalar curvature invariant is a sum of Weyl invariants, we may put

$$
f_{i}=\sum_{\ell_{i}=1}^{\mu_{i}} a_{i \ell_{i}} U_{i \ell_{i}}
$$

where the $U_{i \ell_{i}}$ are Weyl invariants. This yields

$$
W=\sum_{\ell_{1}=1}^{\mu_{1}} \cdots \sum_{\ell_{k}=1}^{\mu_{k}} a_{1 \ell_{1}} \cdots a_{k \ell_{k}} U_{1 \ell_{1}} \cdots U_{k \ell_{k}} .
$$

Now, if some of the products $U_{1 \ell_{1}}, \ldots, U_{k \ell_{k}}$ are linearly dependent, then we may always write

$$
W=\sum \cdots \sum \tilde{a}_{1 \ell_{1}} \cdots \tilde{a}_{k \ell_{k}} U_{1 \ell_{1}} \cdots U_{k \ell_{k}}
$$

where we sum here only over the linearly independent products. Then Lemma 3.7 implies that each of these products is an additive Weyl invariant, and hence all the products in (3.4) are also additive. Then Lemma 3.8 implies that each of these products can only contain one factor, and so $\mu_{1}=\mu_{2}=\cdots=\mu_{k}=k=1$.

From these lemmas we obtain

Corollary 3.10. Let $W$ be an additive Weyl invariant. Then all the possible polynomials $p$ in $W \circ \pi=p \circ \phi_{m}$ are irreducible.

Proof. Put $p=p_{1} \cdots p_{k}$, where each $p_{i}, i=1, \ldots, k$, is irreducible, and $p \circ \phi_{m}=$ $\left(p_{1} \circ \phi_{m}\right) \cdots\left(p_{k} \circ \phi_{m}\right)=\left(f_{1} \circ \pi\right) \cdots\left(f_{k} \circ \pi\right)$. Then $W=f_{1} \cdots f_{k}$, and Lemma 3.9 yields $k=1$, which proves the required result.

\section{Commutativity of invariant differential operators}

Before giving an application of these results we start with some motivating considerations. Let $q \in(M, g)$ and let $\xi$ be a unit vector of $T_{q} M . \gamma: t \mapsto \exp _{m}(t \xi)$ denotes the geodesic through $q$ with tangent vector $\xi$ at $q$. We always suppose $t<i(q)$, where $i(q)$ denotes the injectivity radius at $q$. Now, let $\left\{e_{1}, \ldots, e_{n}\right\}$ be an orthonormal basis of $T_{q} M$ and $\left(x_{1}, \ldots, x_{n}\right)$ the corresponding system of normal coordinates centered at $q$ and with $\frac{\partial}{\partial x_{i}}(q)=e_{i}, i=1, \ldots, n$. Further, let $\theta_{q}$ be the volume density function of $\exp _{q}$ given by

$$
\theta_{q}=\left(\operatorname{det}\left(g_{i j}\right)\right)^{1 / 2},
$$

where $g_{i j}=g\left(\frac{\partial}{\partial x_{i}}, \frac{\partial}{\partial x_{j}}\right)$. Then the local geodesic symmetry $s_{q}: \exp _{q}(t \xi) \mapsto$ $\exp _{q}(-t \xi)$ is a volume-preserving (up to sign) local diffeomorphism if and only if

$$
\theta_{q}\left(\exp _{q}(t \xi)\right)=\theta_{q}\left(\exp _{q}(-t \xi)\right)
$$

for all unit $\xi \in T_{q} M$ and all $q \in M$. The study of these spaces has been initiated in [6], and such spaces have been called D'Atri spaces [23]. We refer to [11] for an extensive survey. They may also be characterized by using a commutativity relation for some special differential operators. More precisely, define a local differential operator $\bar{\Delta}_{q}$ by

$$
\bar{\Delta}_{q} f=\sum_{i=1}^{n} \frac{\partial^{2} f}{\partial x_{i}^{2}}
$$


and put

$$
\tilde{\Delta}^{(k)} f(q)=\left(\bar{\Delta}_{q}\right)^{k} f(q) .
$$

Then $\tilde{\Delta}^{(k)}$ is a global differential operator on $M$, called the Euclidean Laplacian of $\operatorname{order} 2 k[7]$. They can be expressed as a function of $\Delta^{k}$, the curvature tensor and its covariant derivatives (see [7] for more details). We have [20]: a) $(M, g)$ is a D'Atri space if the Laplacian $\Delta$ commutes with all $\tilde{\Delta}^{(k)}, k \in \mathbb{N}$, and b) $(M, g)$ is a D'Atri space if and only if $\Delta$ commutes with all differential operators $f \mapsto \tilde{\Delta}^{(k)}\left(\theta_{q} f\right)$. (See also [16].)

Now we prove

Theorem 4.1. Let $(M, g)$ be a Riemannian manifold such that the Laplacian $\Delta$ commutes with all second order differential operators $L=\eta^{i j} \nabla_{i j}^{2}$, where $\eta$ is a tensor field such that $\eta^{i j}$ are polynomials in the components of $R$ and its covariant derivatives and such that $\alpha=\eta_{i}^{i}$ is an additive Weyl invariant. Then $(M, g)$ is locally homogeneous.

Proof. Following [19], $L \Delta=\Delta L$ implies $\nabla_{i j}^{2} \alpha=0$ and moreover, if $M$ is locally irreducible, then $\alpha$ is a global constant. Hence the result follows from Corollary 3.4. Next, let $M$ be locally reducible and put $M=M_{1} \times \cdots \times M_{r}$. Then $\nabla_{A B}^{2} \alpha=\nabla_{A B}^{2}\left(\alpha_{1}+\cdots+\alpha_{r}\right)$ since $\alpha=\alpha_{1}+\cdots+\alpha_{r}$, where $\alpha_{i}, i=1, \ldots, r$, are the corresponding Weyl invariants on $M_{i}$. This implies $\nabla_{a b}^{2} \alpha_{i}=0$, where $a, b$ are indices on $M_{i}$. Since $M_{i}$ is irreducible, we get that $\alpha_{i}$ is constant on $M_{i}, i=1, \ldots, r$, and so $\alpha$ is a global constant. Hence, the result follows again from Corollary 3.4.

We note that, given an additive Weyl invariant $\alpha$, then $L=\frac{1}{n} \alpha g^{i j} \nabla_{i j}^{2}=\frac{\alpha}{n} \Delta$ is a second order differential operator as in Theorem 4.1, although there are several other possibilities as one can see immediately from (3.2). One just deletes one of the $\delta$ in the summation to produce other examples. By using a similar proof one then obtains

Corollary 4.2. Let $(M, g)$ be a Riemannian manifold such that the Laplacian $\Delta$ commutes with all differential operators $\alpha \Delta$, where $\alpha$ is an additive Weyl invariant. Then $(M, g)$ is locally homogeneous.

Further, we have

Corollary 4.3. Let $(M, g)$ be a Riemannian manifold and denote by $\operatorname{Isom}_{\text {loc }}(M)$ the pseudogroup of local isometries of $M$. Further, let $\mathcal{D}_{\text {loc }}(M)$ denote the algebra of all $\operatorname{Isom}_{\text {loc }}(M, g)$-invariant differential operators. Let $\mathcal{Z}$ be its center. Then if $\Delta \in \mathcal{Z}$ we have

(i) $(M, g)$ is locally homogeneous;

(ii) $(M, g)$ is a D'Atri space;

(iii) $(M, g)$ is a $\mathcal{C}$-space.

Here a $\mathcal{C}$-space is an $(M, g)$ such that the eigenvalues of the Jacobi operators $R_{\gamma^{\prime} \cdot \gamma^{\prime}}$ are constant along the unit speed geodesic $\gamma$ for each $\gamma$ [4].

Proof. (i) follows from Theorem 4.1. (ii) follows from [16], [20] because the Euclidean Laplacians $\tilde{\Delta}^{(k)}$ (and also the operators $\tilde{\Delta}^{(k)}\left(\theta_{q} \cdot\right)$ ) belong to $\mathcal{D}_{l o c}$. Finally, (iii) follows from a result of Sumitomo as in [4]. 
Corollary 4.4. Let $(M, g)$ be a four-dimensional Einstein space with the property that $\Delta$ commutes with all second order operators as in Theorem 4.1 (or Corollary 4.2), where $\alpha$ is an additive Weyl invariant of order $m+1 \leq 6$. Then $(M, g)$ is locally symmetric.

Proof. It follows from Theorem 4.1 (or Corollary 4.2) and Theorem 2.3 that $(M, g)$ is locally homogeneous. Then a well-known result of Jensen implies that $(M, g)$ is locally symmetric.

A commutative (in the broader sense) Riemannian manifold is a homogeneous $(M, g)$ such that $\mathcal{D}$ is a commutative algebra (see [3], [11] for more details). Hence we have

Corollary 4.5. A complete, simply connected $(M, g)$ such that $\mathcal{D}$ is a commutative algebra is a homogeneous space.

Moreover, we have

Corollary 4.6. Let $(M, g)$ be a complete, simply connected Riemannian manifold of dimension 2 or 3 or an Einstein space when $\operatorname{dim} M=4$. Then $(M, g)$ is a commutative space if and only if $\Delta$ belongs to the center of $\mathcal{D}$.

Proof. First we note that two- or three-dimensional, complete, simply connected spaces are D'Atri spaces if and only if they are commutative [6], [9]. Then, the required result follows from this and Corollary 4.3. For four-dimensional Einstein spaces the result follows at once from Corollary 4.4 since symmetric spaces are commutative spaces.

Note that $(M, g)$ is locally isometric to a commutative space in Corollary 4.6 when we delete the words "complete" and "simply connected" in the hypothesis.

We finish with

Corollary 4.7. There exist D'Atri spaces such that $\Delta \notin \mathcal{Z}$.

Proof. The non-symmetric harmonic spaces discovered by Damek and Ricci [5] are D'Atri spaces but are not $\mathcal{C}$-spaces [2], [3]. Hence, Corollary 4.3 implies that $\Delta$ does not belong to $\mathcal{Z}$.

\section{REFERENCES}

1. M. Berger, P. Gauduchon and E. Mazet, Le spectre d'une variété riemannienne, Lecture Notes in Math. 194, Springer-Verlag, Berlin, Heidelberg, New York, 1971. MR 43:8025

2. J. Berndt, F. Tricerri and L. Vanhecke, Geometry of generalized Heisenberg groups and their Damek-Ricci harmonic extensions, C. R. Acad. Sci. Paris Sér. I Math. 318 (1994), 471-476. MR 95a:53074

3. J. Berndt, F. Tricerri and L. Vanhecke, Generalized Heisenberg groups and Damek-Ricci harmonic spaces, Lecture Notes in Math. 1598, Springer-Verlag, Berlin, Heidelberg, New York, 1995. CMP 1995:15

4. J. Berndt and L. Vanhecke, Two natural generalizations of locally symmetric spaces, Diff. Geom. Appl. 2 (1992), 57-80. MR 94j:53061

5. E. Damek and F. Ricci, A class of nonsymmetric harmonic Riemannian spaces, Bull. Amer. Math. Soc. (N.S.) 27 (1992), 139-142. MR 93b:53043

6. J. E. D'Atri and H. K. Nickerson, Divergence-preserving geodesic symmetries, J. Differential Geom. 3 (1969), 467-476. MR 41:7574

7. A. Gray and T. J. Willmore, Mean-value theorems for Riemannian manifolds, Proc. Roy. Soc. Edinburgh Sect. A 92 (1982), 343-364. MR 84f:53038 
8. M. Gromov, Partial Differential Relations, Ergeb. Math. Grenzgeb. 3. Folge 9, SpringerVerlag, Berlin, Heidelberg, New York, 1986. MR 90a:58201

9. O. Kowalski, Spaces with volume-preserving symmetries and related classes of Riemannian manifolds, Rend. Sem. Mat. Univ. Politec. Torino, Fascicolo Speciale Settembre, 1983, pp. 223232.

10. O. Kowalski, Counter-example to the "Second Singer's Theorem", Ann. Global Anal. Geom. 8 (1990), 211-214. MR 92b:53057

11. O. Kowalski, F. Prüfer and L. Vanhecke, D'Atri spaces, preprint, 1995.

12. O. Kowalski and L. Vanhecke, Ball-homogeneous and disk-homogeneous Riemannian manifolds, Math. Z. 180 (1982), 429-444. MR 84c:53043

13. L. Nicolodi and F. Tricerri, On two theorems of I.M. Singer about homogeneous spaces, Ann. Global Anal. Geom. 8 (1990), 193-209. MR 92b:53075

14. V. Poenaru, Singularités $C^{\infty}$ en présence de symétrie, Lecture Notes in Math. 510, SpringerVerlag, Berlin, Heidelberg, New York, 1976. MR 55:13471

15. C. Procesi and G. Schwarz, Inequalities defining orbit spaces, Inv. Math. 81 (1985), 539-554. MR 87h:20078

16. F. Prüfer, On compact Riemannian manifolds with volume-preserving symmetries, Ann. Global Anal. Geom. 7 (1989), 133-140. MR 91c:53047

17. I.M. Singer, Infinitesimally homogeneous spaces, Comm. Pure Appl. Math. 13 (1960), 685697. MR 24:A1100

18. A. Spiro, Lie pseudogroups and locally homogeneous Riemannian spaces, Boll. Un. Mat. Ital. B 6 (1992), 843-872. MR 94a:53085

19. T. Sumitomo, On the commutator of differential operators, Hokkaido Math. J. 1 (1972), 30-42. MR 47:5785

20. Z.I. Szabó, Spectral theory for operator families on Riemannian manifolds, Differential geometry, Proc. Summer Research Institute on Differential Geometry, University of California, Los Angeles, 1990, Proc. Sympos. Pure Math., 54, Part 3 (R. Greene and S.T. Yau, eds.), 1993, pp. 615-665. MR 94k:58152

21. F. Tricerri, Locally homogeneous Riemannian manifolds, Rend. Sem. Mat. Univ. Politec. Torino 50 (1992), 411-426. MR 94k:53065

22. L. Vanhecke, Geometry in normal and tubular neighborhoods, Proc. Workshop on Differential Geometry and Topology Cala Gonone (Sardinia) 1988, Rend. Sem. Fac. Sci. Univ. Cagliari, Supplemento al vol. 58 (1988), 73-176. MR 92g:53037

23. L. Vanhecke and T. J. Willmore, Interaction of tubes and spheres, Math. Ann. 263 (1983), 31-42. MR 85c:53085

24. H. Weyl, The classical groups, their invariants and representations, Princeton Univ. Press, 1946. MR 1:42c

Universität Leipzig, Fakultät für Mathematik und Informatik, Mathematisches INStitut, Augustusplatz 10, D-04109, Leipzig, Germany

E-mail address: pruefer@mathematik.uni-leipzig.d400.de

Katholieke Universiteit leuven, Departement of Mathematics, Celestijnenlaan 200B, B-3001 Leuven, Belgium

E-mail address: lieven.vanhecke@wis.kuleuven.ac.be 